\title{
ERRATA IN THEOREMS BY TOMLINSON FORT
}

\author{
JACKIE B. GARNER
}

In the April 1958 issue of these Proceedings, Tomlinson Fort presents two theorems on difference and differential equations [1]. The proof of Theorem I is incorrect; the argument went wrong on page 290 , line 19 . The hypotheses of the theorem do not assure the solution of

$$
y_{p_{2}+1}\left(k_{1}\right), \cdots, y_{n}\left(k_{1}\right)
$$

in terms of

$$
y_{p_{2}+1}\left(k_{2}\right), \cdots, y_{n}\left(k_{2}\right) \text {. }
$$

This is readily seen for the system

$$
\begin{array}{cc}
y_{\nu}(i+1)=\sum_{\mu=1}^{3} a_{\nu \mu}(i) y_{\mu}(i), \quad \nu=1,2,3, \\
y_{1}(0)=G_{1}, \quad y_{2}(1)=G_{2}, \quad y_{3}(2)=G_{3} .
\end{array}
$$

The proof of Theorem II depends on Theorem I. Even if Theorem I were valid, the hypotheses

(i) $g_{\nu \mu}(x)$ are continuous,

(ii) $D_{n-p_{j}}(x, \alpha, \beta)>0, k_{j-1} \leqq x<k$,

of Theorem II are contradictory whenever there are more than two distinct $k_{i}$. For example, (ii) requires $g_{13}(x)$ and $g_{31}(x)$ to be discontinuous at $x=k_{1}$ for the system

$$
\begin{aligned}
\frac{d y_{\nu}}{d x} & =\sum_{\mu=1}^{3} g_{\nu \mu}(x) y_{\nu}, & \\
y_{\nu}\left(k_{\nu-1}\right) & =G_{\nu}, & \nu=1,2,3,
\end{aligned}
$$

when $k_{0}<k_{1}<k_{2}$.

\section{REFERENCE}

1. Tomlinson Fort, Linear difference and differential equations satisfying conditions at more than one point, Proc. Amer. Math. Soc. vol. 9 (1958) pp. 287-292.

AUBURN UNIVERSITY AND

Louisiana Polytechnic Institute

Received by the editors June 13, 1960 and, in revised form, July 16, 1960. 